\title{
Interferometric measurement of local spin fluctuations in a quantum gas
}

\author{
Jakob Meineke ${ }^{1}$, Jean-Philippe Brantut ${ }^{1}$, David Stadler ${ }^{1}$, Torben Müller ${ }^{1}$, Henning Moritz ${ }^{2}$ and \\ Tilman Esslinger ${ }^{1 \star}$
}

\begin{abstract}
Ultracold gases provide a controlled environment that is ideal for studying many intriguing phenomena associated with quantum correlated systems ${ }^{1}$. Current efforts are directed towards the identification of magnetic properties ${ }^{2-4}$, as well as the creation and detection of exotic quantum phases ${ }^{5-7}$. In this context, a mapping of the spin polarization of the atoms to the state of a single-mode light beam has been proposed ${ }^{8}$. Here we introduce a quantum-limited interferometer that realizes such an atom-light interface ${ }^{9}$ with high spatial resolution. We measure the probability distribution of the local spin polarization in a trapped Fermi gas, showing a reduction of spin fluctuations by up to $4.6(3) \mathrm{dB}$ below shot noise in weakly interacting Fermi gases, and by $9.4(8) \mathrm{dB}$ for strong interactions. We deduce the magnetic susceptibility as a function of temperature and discuss our measurements in terms of an entanglement witness.
\end{abstract}

Quantum mechanics manifests itself in the correlations between the constituent parts of a physical system. These correlations quantify the probability of joint measurements, and are experimentally observable in the statistical distribution of the outcomes of repeated measurements. Experiments studying density fluctuations have successfully demonstrated the potential of these techniques as a tool to study local thermodynamic properties of quantum gases $^{10-13}$. In another context, interferometric methods have been used to study spin fluctuations in atomic vapours, leading to the observation of entanglement and spin squeezing ${ }^{9,14,15}$. More recently, several authors have proposed applying similar techniques to map quantum fluctuations, generated by the many-body dynamics in a quantum gas $^{6-8}$, on to the optical field of a single-mode probe beam. In a different approach, speckle noise originating from out-of-focus regions in off-resonant imaging was related to the spin fluctuations of a Fermi gas ${ }^{16}$. In this Letter, we use a shot-noise-limited interferometer to directly measure the probability distribution of the local spin fluctuations in a two-component quantum degenerate Fermi gas.

Our interferometer is analogous to a Young's double slit experiment. Two tightly focused beams, the probe and the local oscillator, are focused to separate points, as shown in Fig. 1a, and overlap in the far-field. The position and visibility of the resulting interference pattern are determined by changes in the phase and amplitude of the probe beam, which passes through an atomic cloud whereas the local oscillator does not. The analysis of the interference pattern thus allows the reconstruction of both quadratures of the probe beam, phase and amplitude, which carry information about the local properties of the atomic cloud.
A probe beam passing through a mixture of ${ }^{6} \mathrm{Li}$ atoms in the lowest two hyperfine states $|1\rangle$ and $|2\rangle$ acquires a phase shift given by

$$
\phi=\sigma_{0}\left(\frac{n_{1} \delta_{1}}{1+s+4 \delta_{1}^{2}}+\frac{n_{2} \delta_{2}}{1+s+4 \delta_{2}^{2}}\right)
$$

with $\sigma_{0}$ the resonant scattering cross-section, $s$ the saturation parameter, and $n_{i}$ and $\delta_{i}$ the line-of-sight integrated density and the frequency detuning in atomic linewidths for state $|i\rangle$, respectively. By choosing the detuning exactly in between the two resonances, $\delta_{1}=-\delta_{2}=6.4$, the phase shift $\phi$ is proportional to the line-ofsight integrated spin polarization density $m=n_{1}-n_{2}$. A single measurement of the phase shift yields the spin polarization of the given experimental realization. Consequently, the full probability distribution of the spin polarization can be reconstructed from repeated measurements.

To validate our procedure we first show that equation (1) holds in the parameter regime of the experiment and, second, that the phase measurement is only limited by photon shot noise of the probe beam. Then, each additionally detected photon leads to a projection of the atomic state into a smaller subspace. To verify the first point, we measure the frequency-dependent phase shift and optical density for a Fermi gas comprised of atoms in state $|2\rangle$ only, see Methods for preparation and data-processing. Figure $2 \mathrm{a}$ shows the characteristic asymmetric profile of the phase whereas the optical density is described well by a Lorentzian. The solid lines result from fitting the phase data to equation (1) with $n_{1}=0$ and agree with the measurement provided the probe duration is $\sim 1 \mu \mathrm{s}$ and the saturation is less than $\sim 10$. The use of stronger (longer) pulses leads to a systematic shift of measured phases to larger values, owing to the light forces resulting from the strong focusing of the probe beam and scattering of photons. To verify the second point, we measure the phase variance as a function of the photon number, as shown in Fig. 2b. From the power-law behaviour of the phase variance we deduce that photon shot noise limits the sensitivity of the phase measurement. Indeed, the phase noise is expected to be given by $\delta \phi^{2}=1 / \eta N$ (ref. 17), where $N$ is the number of photons and $\eta=0.6$ the quantum efficiency, which is determined in an independent measurement.

We first measure the distribution of the spin polarization for weakly interacting Fermi gases at three different temperatures given by $T_{1}=8.5(5) \mu \mathrm{K}, T_{2}=2.0(2) \mu \mathrm{K}$ and $T_{3}=0.58(5) \mu \mathrm{K}$, see Methods for preparation. Figure 3a shows that the probability distributions of the spin polarization have a narrower width the lower the temperature of the gas. The distributions exhibit no significant asymmetry and are well described by Gaussian functions, as expected from

\footnotetext{
${ }^{1}$ Institute for Quantum Electronics, ETH Zurich, 8093 Zurich, Switzerland, ${ }^{2}$ Institut für Laser-Physik, Universität Hamburg, 22761 Hamburg, Germany. ^e-mail: esslinger@phys.ethz.ch.
} 

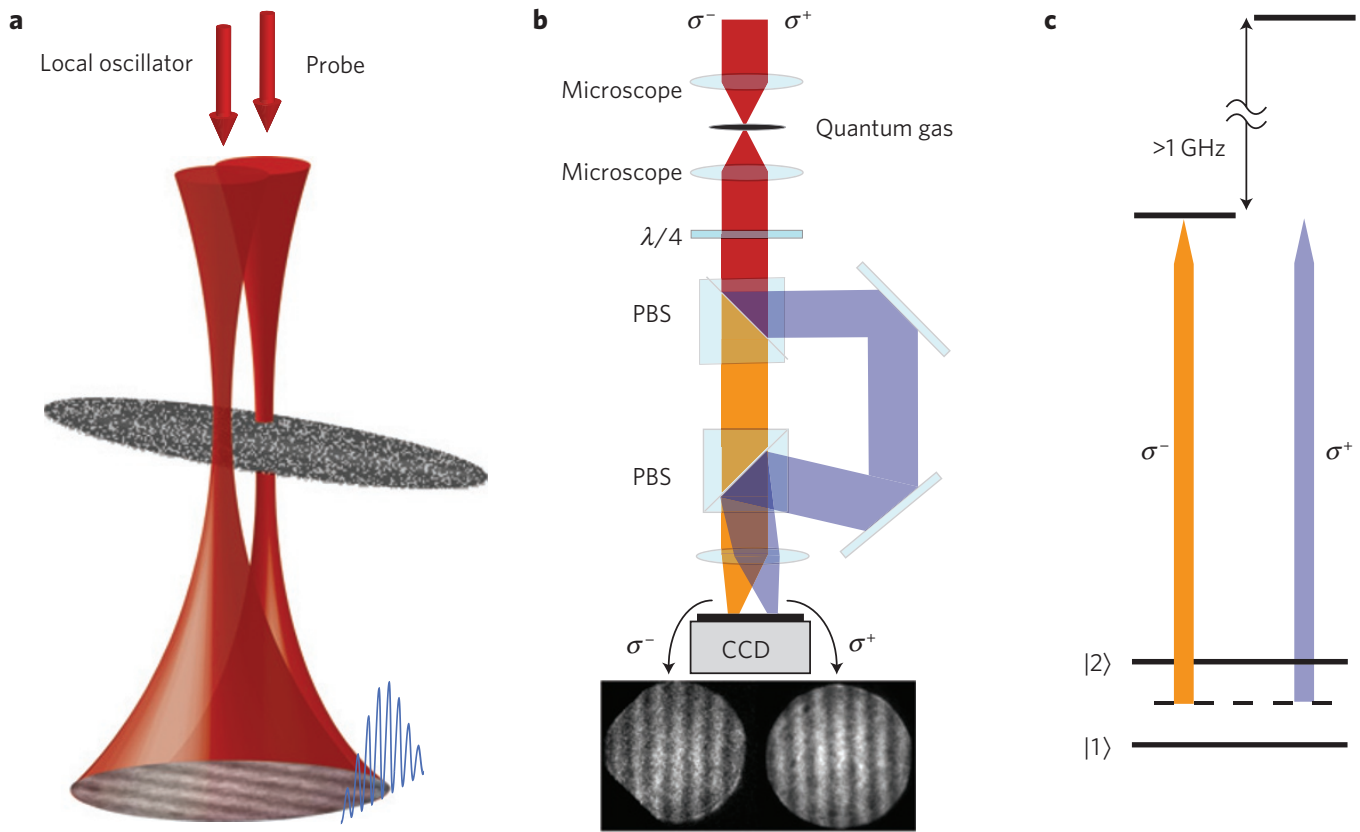

Figure 1 | Interferometer set-up. a, Interferometer beams in the vicinity of the atomic cloud. The probe passes through the cloud shown in grey, whereas the local oscillator passes by the side of it. The beams (minimum $1 / e^{2}$-radius $1.2 \mu \mathrm{m}$ ) overlap in the far-field, giving rise to an interference pattern as shown. See Methods for creation of the interferometer beams. $\mathbf{b}$, Optical set-up to obtain two interference patterns, only one of which is affected by the atoms. Using a quarter-wave retardation plate $(\lambda / 4)$ and two polarizing beam splitters (PBS), the $\sigma^{-}$-component of the polarization, which interacts with the atoms, is separated from the $\sigma^{+}$-component. This yields two far-field interference patterns on one image, as shown in the lower part of the figure (see Methods). The lens adjusts the size of the patterns on the camera. c, Level-scheme illustrating that only $\sigma^{-}$light interacts with the atoms. $\sigma^{+}$light is detuned far from resonance. Transitions from both states to their respective excited states are nearly closed cycling transitions.

the large number of atoms in the probe beam, $\sim 500$ in each state. For weakly interacting Fermi gases, number fluctuations in each hyperfine state are independent, so that fluctuations of the spin polarization are given by $\delta m^{2}=\delta n_{1}^{2}+\delta n_{2}^{2}$. Consequently, with the onset of quantum degeneracy, spin fluctuations are reduced, as in each state only atoms at the Fermi edge contribute to the fluctuations, which is a manifestation of antibunching due to Pauli's principle ${ }^{12,13}$. The measured variances of the spin polarization, in order of decreasing temperature, are $\delta m_{T_{1}}^{2}=35(6) \mu \mathrm{m}^{-4}$, $\delta m_{T_{2}}^{2}=27(3) \mu \mathrm{m}^{-4}$ and $\delta m_{T_{3}}^{2}=15(3) \mu \mathrm{m}^{-4}$. Here, the background corresponding to a standard deviation of $\pm 13(2)$ atoms in the probed volume has been subtracted (see Methods). The column density in the probed region was $n_{1}=n_{2}=n_{\text {col }} / 2=110 \mu \mathrm{m}^{-2}$ for the gases at $T_{2}$ and $T_{3}$ and $20 \%$ lower for the gas at $T_{1}$. From our measurement we determine a reduction of the spin fluctuations as compared with a thermal gas prepared with the same column density by 2.1(2) dB for the gas at $T_{2}$ and $4.6(3) \mathrm{dB}$ for the gas at $T_{3}$, as shown in Fig. 3b, which is in quantitative agreement with theory for a non-interacting Fermi gas (see Methods).

We now turn to the study of a gas with strong repulsive interactions, prepared close to a Feshbach resonance at temperature $T_{\text {mol }}=0.36(10) \mu \mathrm{K}$ (see Methods). The resulting histogram is displayed in Fig. $3 \mathrm{a}$ and shows a distribution of the spin polarization that is significantly narrower than for the weakly interacting gases. This reflects the fact that, for interacting gases, correlations are present between the different spin states. In particular, for strong repulsive interactions close to a Feshbach resonance, weakly bound molecules form ${ }^{1}$ and the number of atoms in the two states will always be equal. Spin fluctuations in the atom number difference are created at the cost of breaking molecules and are consequently suppressed. We measure $\delta m_{\mathrm{mol}}^{2}=5(2) \mu \mathrm{m}^{-4}$ at $n_{\mathrm{col}} / 2=110 \mu \mathrm{m}^{-2}$, with the background subtracted as before. This corresponds to a reduction by $9.4(8) \mathrm{dB}$ as compared with a non-interacting thermal gas. A reduction by $\sim 18 \mathrm{~dB}$ is expected from ref. 8 for a molecular BEC at zero temperature. The observation of a lower value could be caused by pair-breaking or fluctuations of the probe frequency (see Methods).

The measured values for the spin fluctuations can be used to determine the magnetic susceptibility $\chi$ through the fluctuationdissipation theorem (FDT; ref. 18,19), provided the probed system is in grand-canonical equilibrium with its surroundings. Because of column integration, the FDT here reads $k T \int \chi=k T \chi_{\text {col }}=A \delta m^{2}$, where $\chi_{\text {col }}$ is the column-integrated magnetic susceptibility, $k$ Boltzmann's constant and $A$ the effective area of the probed column. For small volumes and at low temperatures corrections to the FDT are expected, because correlations between the probed system and its surroundings cannot be neglected ${ }^{20,21}$. Using ref. 20 and including column integration, we estimate the corrections to be less than $10 \%$, even for our coldest samples. Figure $3 \mathrm{c}$ shows the column-integrated magnetic susceptibility per particle, $\chi_{\mathrm{col}} / n_{\mathrm{col}}$, as a function of temperature. It relates the spin imbalance to the energy needed to create it. For high temperatures, the susceptibility is inversely proportional to the temperature, as expected. For low temperatures and with the onset of quantum degeneracy, the susceptibility saturates to a value depending on the trap details: the stiffer the trap, the lower the susceptibility. The solid line shows theory for non-interacting fermions calculated for the trap parameters of the gas at $T_{3}$. The susceptibility for the strongly interacting gas of molecules is lower than for the weakly interacting gas at $T_{3}$, despite its lower temperature and weaker trap.

The link between the magnetic susceptibility and spin fluctuations has been proposed as a macroscopic entanglement witness in solid state systems ${ }^{22}$. We now apply this concept to our measurement of the spin fluctuations. For this purpose we describe each particle as a two-level system, which together form an effective spin $\mathbf{M}$ (refs 22,23), where $\left\langle M_{z}\right\rangle$ is proportional to the atom number difference and $\delta M_{z}^{2}=A^{2} \delta m^{2}$. For all separable states, the ratio of the spin fluctuations to the total atom number is bounded from 


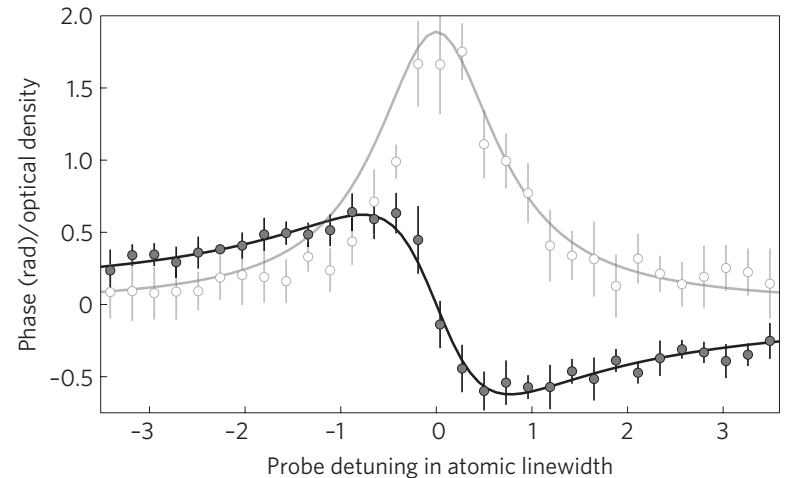

b

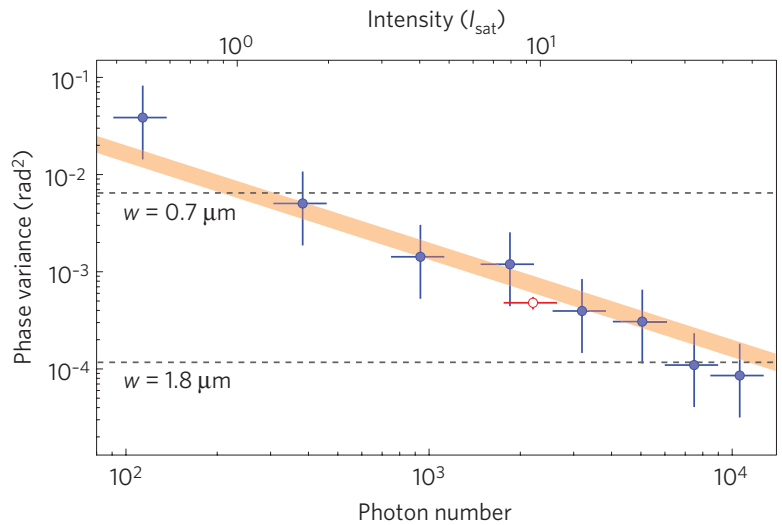

Figure 2 | Interferometer performance. a, Measured phase (grey filled circle) and optical density (open circle) as a function of detuning. Maximum saturation of the probe beam $s=1.2$, duration of the probe pulses $1.2 \mu \mathrm{s}$. Solid lines result from a fit to the phase data using the model described in the text, yielding $n_{2} \sigma_{0}=3.2$, effective saturation 0.6 and an effective linewidth that is $20 \%$ broader than the natural linewidth of 5.9 MHz, which we attribute to the probe laser. Error bars show standard deviations. b. Measured phase variance (without atoms) $\delta \phi^{2}$ (blue filled circle) as a function of photon number in the probe beam determined from 100 measurements for each point. The empty circle (red open circle) indicates the phase variance for the intensity at which the spin polarization measurements were made. Error bars are an uncorrelated sum of statistical and systematic uncertainties. Expected phase noise (indicated by the orange band) for quantum efficiency $\eta=0.6$. The width of the line corresponds to $20 \%$ errors estimated from the uncertainty of our determination of $\eta$. The dashed lines indicate the square of the phase shift expected for a single atom fixed in space at a detuning of half the atomic linewidth for the indicated $1 / e^{2}$-waists of the probe beam ${ }^{32}$.

below. For a system in grand-canonical equilibrium described by a Hamiltonian that is invariant under rotations of the spin state, this bound is expressed by the inequality

$$
\frac{A \delta m^{2}}{n_{\mathrm{col}}} \geq \frac{2}{3}
$$

(see Methods and Supplementary Information for details). Figure $3 \mathrm{~d}$ shows the spin fluctuations $A \delta m^{2}$ as a function of the column density $n_{\text {col }}$. The gas at $T_{3}$ violates the above inequality, which is expected because the Pauli principle leads to non-separable states at low temperatures ${ }^{24}$. The notion of entanglement applied to indistinguishable particles is the subject of current investigations ${ }^{25}$.

For the case of the strongly interacting gas, we find $A \delta m^{2} / n_{\text {col }}=0.11(4)$, violating the bound by more than 10 standard deviations. The fluctuations are $2.4 \mathrm{~dB}$ lower than expected for a non-interacting gas at the same temperature (bold line in Fig. $3 \mathrm{~d}$ ). This corresponds to $2.4 \mathrm{~dB}$ of spin squeezing, following ref. 26 .
Our analysis uses the following assumptions. (1) We create a gas with equal atom number in the two states, which leads to $\left\langle M_{z}\right\rangle=0$. (2) We probe the system in thermal equilibrium. As a consequence, the transverse components have dephased to the amount permitted by Pauli's principle and we have $\left\langle M_{x}\right\rangle=\left\langle M_{y}\right\rangle=0$. (3) The time evolution is described by a Hamiltonian that is invariant under rotations of the $\operatorname{spin}^{27}$. It is therefore sufficient to measure only the $z$-component.

The rigorous application of the entanglement witness ${ }^{22,23}$ requires equal coupling of all atoms to the probe beam. Here, we take into account the inhomogeneity of the probe beam by introducing the effective area $A$.

In conclusion, we have measured the probability distribution of the spin fluctuations in a trapped Fermi gas. We have discussed our measurement in terms of an entanglement witness. Our work constitutes a first step towards the detection of entanglement in many-body states in quantum gases. The detection of higher-order correlations could be achieved by extracting higher moments of the probability distribution for the spin polarization ${ }^{28}$.

\section{Methods}

Generation of interferometer beams. The interferometer beams are generated by applying two radio frequencies differing by $20 \mathrm{MHz}$ along each of the axes of a two-axis acousto-optical deflector (AOD), very similar to previous work ${ }^{29}$. This results in four beams in the $-1 /-1$ diffraction order of the AOD which, after passing through a high-resolution microscope objective, are arranged in a square. Two of these beams, the probe beam and local oscillator, have exactly the same frequency and form the interference pattern on the camera. The other two beams are detuned by $\pm 20 \mathrm{MHz}$ and their interference patterns average out over the duration of the probe pulses. The intensities of the beams are controlled by the power in the individual radio frequencies so that the local oscillator is 20 times as intense as the probe beam. Phase stability is ensured by deriving each radio frequency from the same source for both axes. The light beams are elliptically polarized. Owing to the birefringence of the atomic cloud in a magnetic field, also used in polarization-contrast imaging ${ }^{30}$, only the $\sigma^{-}$-polarized component of the light interacts with the atoms, whereas the $\sigma^{+}$- component passes undisturbed (Fig. 1b). The power ratio of $\sigma^{+}$- and $\sigma^{-}$-component is about 10 .

Experimental sequence. An equal mixture of ${ }^{6} \mathrm{Li}$ atoms in the two lowest hyperfine states, denoted by $|1\rangle=\left|m_{\mathrm{J}}=-1 / 2, m_{\mathrm{I}}=1\right\rangle$ and $|2\rangle=\left|m_{\mathrm{J}}=-1 / 2, m_{\mathrm{I}}=0\right\rangle$ is prepared, similar to previous work ${ }^{12}$. A second dipole trap with a wavelength of $767 \mathrm{~nm}$ and a $1 / e^{2}$-radius of $10 \mu \mathrm{m}$ is then switched slowly on over $500 \mathrm{~ms}$ and is used to locally increase the total column density. Finally, the magnetic field is ramped to $475 \mathrm{G}$, where the scattering length is $a=-100 a_{0}$, with $a_{0}$ the Bohr radius. The final trap depths of the large dipole trap are $84 \mu \mathrm{K}$ for $T_{1}, 19 \mu \mathrm{K}$ for $T_{2}$ and $10 \mu \mathrm{K}$ for $T_{3}$, corresponding to $1,050 \mathrm{~mW}, 235 \mathrm{~mW}$ and $130 \mathrm{~mW}$, respectively. The trap depths of the second dipole trap are $9 \mu \mathrm{K}, 4.5 \mu \mathrm{K}$ and $1.5 \mu \mathrm{K}$, respectively. The central region of the cloud is probed interferometrically with a pulse of $1.2 \mu$ s duration at a maximum saturation of 9 . Experiments are repeated 400 times. Images of the whole cloud are taken after the interferometric measurement to determine the total atom number as well as the temperature from the radial expansion after $1 \mathrm{~ms}$ time-of-flight. Experiments that show a larger deviation of the total atom number than $5 \%$ are discarded, amounting to $10-20 \%$ of the images. For the preparation of the strongly interacting gas, we ramp directly to $800 \mathrm{G}$, where $a=7,000 a_{0}$ and the trap depth is lowered to $17 \mathrm{~mW}$, followed by recompression to $30 \mathrm{~mW}$, which corresponds to a trap depth of $4.8 \mu \mathrm{K}$ for the molecules. The experiment is repeated 200 times. We estimate the final temperature to be one tenth of the relevant trap depth, leading to $T_{\mathrm{mol}}=0.36(10) \mu \mathrm{K}$ after recompression. For the preparation of a gas containing only atoms in state $|2\rangle$, we hold the gas for $100 \mathrm{~ms}$ close to a $p$-wave Feshbach resonance at $159 \mathrm{G}$, which leads to the loss of nearly all particles in state $|1\rangle$. Subsequent evaporation of the remaining atoms leads to a non-degenerate gas of atoms in state $|2\rangle$.

Theory. We compare our measurements for the weakly interacting gases with theory for non-interacting fermions ${ }^{31}$. We determine the temperature and the chemical potential from fits to the time-of-flight images, as in previous work ${ }^{12}$. We then calculate the density distribution in the combined trap, given the fitted temperature and atom number, to determine $T_{\mathrm{F}}$ in the centre of the combined trap. This assumes full thermalization in the presence of the second trap, which is supported by the measured spin fluctuations. The knowledge of $T, \mu$ and the trap shape allows us to calculate the mean and the variance of the atomic density along the line of sight using column integration.

Data processing. Three images are obtained in each experiment, one with atoms in the interferometer and two without. The images are averaged along the direction 


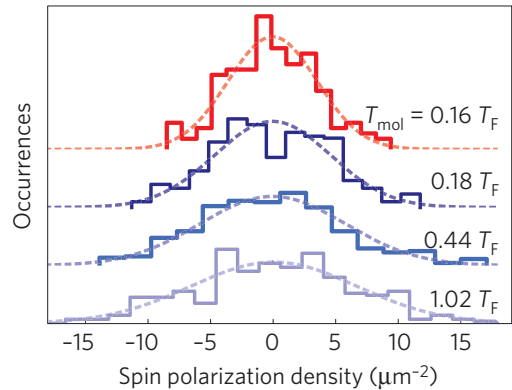

b

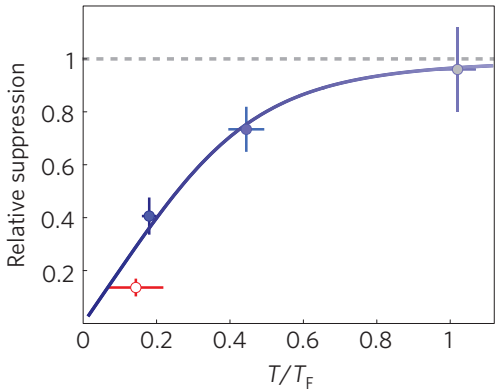

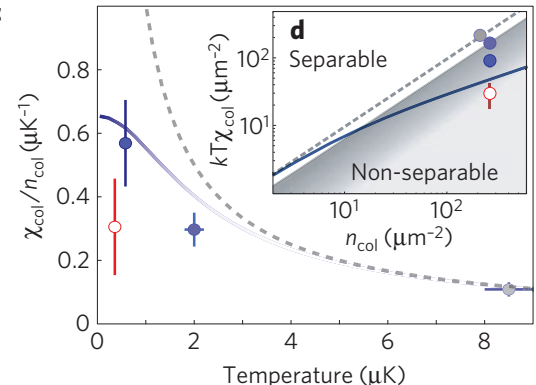

Figure 3 | Spin fluctuations in ultracold Fermi gases. a, Normalized histograms for the measured spin polarization for weakly interacting gases at $T_{1}=1.02(2) T_{\mathrm{F}}$ (light blue line), $T_{2}=0.44(5) T_{\mathrm{F}}$ (blue line) and $T_{3}=0.18(2) T_{\mathrm{F}}$ (dark blue line) as well as for a strongly interacting gas of molecules at $T_{\mathrm{mol}}=0.16(7) T_{\mathrm{F}}$ (red line), where $T_{\mathrm{F}}$ is the Fermi temperature. $\mathbf{b}$, Reduction of spin fluctuations compared with a thermal gas of equal column density as a function of $T / T_{\mathrm{F}}$. Data points are coloured identical to $\mathbf{a}$. The solid line shows theory for a non-interacting Fermi gas. $\mathbf{c}$, Spin susceptibility per atom $\chi_{\mathrm{col}} / n_{\mathrm{col}}$ as a function of temperature for the weakly interacting gas (blue points) and the strongly interacting gas (red open circle). Also shown is the susceptibility for a classical gas (grey dashed line), which varies as $1 / T$, and the theoretical expectation (light blue line) for the trap parameters of the gas at $T_{3}$. d, Spin-fluctuation density $A \delta m^{2}=k T \chi_{\text {col }}$ as a function of column-density $n_{\text {col }}$. The prediction for a thermal gas (grey dashes) is shown along with the boundary separating the separable from the non-separable region. The blue line shows theory for a non-interacting Fermi gas at temperature $T_{\mathrm{mol}}=0.36 \mu \mathrm{K}$. Error bars are an uncorrelated sum of statistical and systematic uncertainties.

parallel to the fringe pattern and a Fourier filter is applied to suppress the low spatial frequencies. For the determination of the mean phase, a simultaneous sinusoidal fit to the $\sigma^{-}$-pattern on images with and without atoms is used. The free parameters are the amplitude and wavelength of the fringe pattern, as well as a common phase for both patterns and a phase-difference for the picture with atoms. Residual mechanical motion, for example due to the microscopes, is compensated for by using the same simultaneous fit applied to the $\sigma^{+}$-pattern. For determination of the phase fluctuations we find it advantageous to analyse the correlations between the $\sigma^{+}$-pattern and the $\sigma^{-}$-pattern on each image. This allows us to exploit the similarity of the two patterns and use the $\sigma^{+}$-pattern to noiselessly amplify the signal contained in the $\sigma^{-}$-pattern, analogous to homodyne techniques. The phase shift due to the atoms causes a displacement of the zero-crossings of the correlation function in the image with atoms as compared with the image without atoms. See Supplementary Informations for further details on data processing.

Determination of effective area. The effective area $A$ relates the spin fluctuations to the mean atomic density and corresponds to the area of a beam with uniform intensity giving the same result. For the nearly non-degenerate gas at $T_{1}=1.02 T_{\mathrm{F}}$, the spin fluctuations are described well by Poissonian statistics because the atoms are uncorrelated. The fluctuations are thus proportional to the average atom number in the probe volume. This allows the determination of the effective area $A=0.97 n_{\mathrm{col}} / \delta m_{\mathrm{T}_{\mathrm{T}}}^{2}=4.9(8) \mu \mathrm{m}^{2}$, corresponding to an effective waist of the probe beam of 1.8(3) $\mu \mathrm{m}$. The factor 0.97 accounts for the residual suppression of the spin fluctuations at $T_{1}=1.02 T_{\mathrm{F}}$.

Background. The contribution to the phase variance originating from photon shot noise is determined from the two images without atoms using the above described procedure. This yields $\delta m_{\mathrm{ber}}^{2}=6.7(1.0) \mu \mathrm{m}^{-4}$ and a standard deviation of the atom number difference in the probed volume of $\sqrt{A^{2} \delta m_{\mathrm{bgr}}^{2}}=13$ (2). Frequency fluctuations of the probe can cause fluctuations in the measured phase. A variance of the probe frequency of $2 \mathrm{MHz}^{2}$ would correspond to apparent spin fluctuations of $5 \mu \mathrm{m}^{-4}$ at the column density in our experiment and could contribute to the difference of our results compared with the theory in ref. 8 .

Entanglement witness. Each atom realizes a two-level system represented by Pauli matrices $\sigma_{x, y, z}$. We define the spin-polarization or magnetization of the probed region as $M_{x, y, z}=\sum_{i} \sigma_{x, y, z}^{i}$, where the sum extends over all atoms in the probe volume. For all separable states the inequality $\delta M_{x}^{2}+\delta M_{y}^{2}+\delta M_{z}^{2} \geq 2\langle N\rangle$ is fulfilled, where $\langle N\rangle$ is the average number of atoms probed ${ }^{22}$. Using the invariance of the Hamiltonian under spin rotations and that we measure expectation values in the grand-canonical ensemble, which show the same symmetry as the Hamiltonian, this reduces to $\delta M_{z}^{2} / N=A \delta m^{2} / n_{\text {col }} \geq 2 / 3$. See Supplementary Information for more details.

Received 11 April 2011; accepted 5 March 2012; published online 8 April 2012

\section{References}

1. Inguscio, M., Ketterle, W. \& Salomon, C. (eds) Ultra-cold Fermi Gases: Proceedings of the International School of Physics 'Enrico Fermi' Vol. 164 (IOS Press, 2007).

2. Jördens, R. et al. Quantitative determination of temperature in the approach to magnetic order of ultracold fermions in an optical lattice. Phys. Rev. Lett. 104, 180401 (2010).
3. Nascimbène, S. et al. Fermi-liquid behavior of the normal phase of a strongly interacting gas of cold atoms. Phys. Rev. Lett. 106, 215303 (2011).

4. Sommer, A., Ku, M., Roati, G. \& Zwierlein, M. W. Universal spin transport in a strongly interacting Fermi gas. Nature 472, 201-204 (2011).

5. Lewenstein, M. et al. Ultracold atoms in optical lattices: Mimicking condensed matter physics and beyond. Adv. Phys. 56, 243-379 (2007).

6. Eckert, K. et al. Quantum non-demolition detection of strongly correlated systems. Nature Phys. 4, 50-54 (2008).

7. Roscilde, T. et al. Quantum polarization spectroscopy of correlations in attractive fermionic gases. New J. Phys. 11, 055041 (2009).

8. Bruun, G. M., Andersen, B. M., Demler, E. \& Sørensen, A. S. Probing spatial spin correlations of ultracold gases by quantum noise spectroscopy. Phys. Rev. Lett. 102, 030401 (2009).

9. Hammerer, K., Sørensen, A. S. \& Polzik, E. S. Quantum interface between light and atomic ensembles. Rev. Mod. Phys. 82, 1041-1093 (2010).

10. Estève, J. et al. Observations of density fluctuations in an elongated Bose gas: Ideal gas and quasicondensate regimes. Phys. Rev. Lett. 96, 130403 (2006).

11. Gemelke, N., Zhang, X., Hung, C. \& Chin, C. In situ observation of incompressible Mott-insulating domains in ultracold atomic gases. Nature 460, 995-998 (2009).

12. Müller, T., Zimmermann, B., Meineke, J., Brantut, J., Esslinger, T. \& Moritz, H. Local observation of antibunching in a trapped Fermi gas. Phys. Rev. Lett. 105, 040401 (2010).

13. Sanner, C. et al. Suppression of density fluctuations in a quantum degenerate Fermi gas. Phys. Rev. Lett. 105, 040402 (2010).

14. Oblak, D. et al. Quantum-noise-limited interferometric measurement of atomic noise: Towards spin squeezing on the Cs clock transition. Phys. Rev. A 71, 043807 (2005).

15. Appel, J. et al. Mesoscopic atomic entanglement for precision measurements beyond the standard quantum limit. Proc. Natl Acad. Sci. USA 106, 10960-10965 (2009).

16. Sanner, C. et al. Speckle imaging of spin fluctuations in a strongly interacting Fermi gas. Phys. Rev. Lett. 106, 010402 (2011).

17. Lye, J. E., Hope, J. J. \& Close, J. D. Nondestructive dynamic detectors for Bose-Einstein condensates. Phys. Rev. A 67, 043609 (2003).

18. Recati, A. \& Stringari, S. Spin fluctuations, susceptibility, and the dipole oscillation of a nearly ferromagnetic Fermi gas. Phys. Rev. Lett. 106, 080402 (2011).

19. Seo, K. \& Sá de Melo, C. A. R. Compressibility and spin susceptibility in the evolution from BCS to BEC superfluids. Preprint at http://arXiv.org/abs/1105.4365 (2011).

20. Klawunn, M., Recati, A., Pitaevskii, L. P. \& Stringari, S. Local atom-number fluctuations in quantum gases at finite temperature. Phys. Rev. A 84, 033612 (2011).

21. Hung, C., Zhang, X., Gemelke, N. \& Chin, C. Observation of scale invariance and universality in two-dimensional Bose gases. Nature 470, 236-239 (2011).

22. Wieśniak, M., Vedral, V. \& Časlav, Brukner. Magnetic susceptibility as a macroscopic entanglement witness. New J. Phys. 7, 258 (2005).

23. Tóth, G., Knapp, C., Gühne, O. \& Briegel, H. J. Spin squeezing and entanglement. Phys. Rev. A 79, 042334 (2009).

24. Vedral, V. Entanglement in the second quantization formalism. Central Eur. J. Phys. 1, 289-306 (2003). 
25. Horodecki, R., Horodecki, P., Horodecki, M. \& Horodecki, K. Quantum entanglement. Rev. Mod. Phys. 81, 865-942 (2009).

26. Kheruntsyan, K. V. Quantum atom optics with fermions from molecular dissociation. Phys. Rev. Lett. 96, 110401 (2006).

27. Zwierlein, M. W., Hadzibabic, Z., Gupta, S. \& Ketterle, W. Spectroscopic insensitivity to cold collisions in a two-state mixture of fermions. Phys. Rev. Lett. 91, 250404 (2003).

28. Cherng, R. W. \& Demler, E. Quantum noise analysis of spin systems realized with cold atoms. New J. Phys. 9, 7 (2007).

29. Zimmermann, B., Müller, T., Meineke, J., Esslinger, T. \& Moritz, H. High-resolution imaging of ultracold fermions in microscopically tailored optical potentials. New J. Phys. 13, 043007 (2011).

30. Bradley, C. C., Sackett, C. A. \& Hulet, R. G. Bose-Einstein condensation of lithium: Observation of limited condensate number. Phys. Rev. Lett. 78, 985-989 (1997).

31. Huang, K. Statistical Mechanics 2nd edn (Wiley, 1987).

32. Aljunid, S. A. et al. Phase shift of a weak coherent beam induced by a single atom. Phys. Rev. Lett. 103, 153601 (2009).

\section{Acknowledgements}

We acknowledge enlightening discussions with M. Christiandl, A. Imamoglu, K. Mølmer, E. Polzik, R. Renner, A. Sørensen, M. Ueda and V. Vuletic and funding from National Centres of Competence in Research (NCCR) MaNep, NCCR QSIT, European Research Council (ERC) SQMS and FP7 FET-open NameQuam. J-P.B. acknowledges the support of the European Union under a Marie Curie IEF fellowship.

\section{Author contributions}

J.M. and J-P.B. analysed the data. J.M., J-P.B., D.S. and T.M. carried out the experimental work. All authors contributed to project planning and to writing the manuscript.

\section{Additional information}

The authors declare no competing financial interests. Supplementary information accompanies this paper on www.nature.com/naturephysics. Reprints and permissions information is available online at www.nature.com/reprints. Correspondence and requests for materials should be addressed to T.E. 